\title{
Características clínicas de usuários abusivos de substâncias psicoativas internados em Unidade de Terapia Intensiva*
}

\author{
Gabriela Botelho Pereira ${ }^{1}$ \\ (D) https://orcid.org/0000-0002-9964-6586 \\ Karine Langmantel Silveira, ${ }^{1,2}$ \\ (D) https://orcid.org/0000-0002-2598-5278 \\ Carla Luciane dos Santos Borges ${ }^{1}$ \\ (1D) https://orcid.org/0000-0003-0535-8768 \\ Michele Mandagará de Oliveira ${ }^{1}$ \\ (D) https://orcid.org/0000-0002-7914-9339
}

Objetivo: descrever as causas de internação e comorbidades de pacientes com diagnóstico de abuso/dependência de substâncias psicoativas internados em Unidade de Terapia Intensiva. Método: estudo retrospectivo, descritivoexploratório, transversal e quantitativo. Resultados: pesquisaram-se 865 prontuários entre 2012 e 2015, e a prevalência de uso de substâncias foi de 51,9\%. Destes, $22 \%$ são usuários de álcool e 48,7\%, de tabaco. Entre os usuários com dependência diagnosticada, as principais causas de internação encontradas foram doença pulmonar obstrutiva crônica (100\%) e hemorragia digestiva (84\%), e a maioria dos pacientes apresentou mais do que uma comorbidade simultaneamente. Conclusão: os resultados apontaram inúmeras causas de internação e comorbidades que demonstraram o comprometimento físico e psíquico que pode estar relacionado ao consumo excessivo de substâncias psicoativas.

Descritores: Transtornos Relacionados ao Uso de Substâncias; Usuários de Drogas; Unidades de Terapia Intensiva; Comorbidade.

\section{Como citar este artigo}

Pereira GB, Silveira KL, Borges CLS, Oliveira MM. Clinical characteristics of drug users hospitalized in an intensive care unit. SMAD, Rev Eletrônica Saúde Mental Álcool Drog. 2020;16(2):1-8. doi:https://dx.doi.org/10.11606/issn.1806-6976.smad.2020.158506 


\section{Clinical characteristics of drug users hospitalized in an intensive care unit}

Objetive: to describe causes of hospitalization and comorbidities of patients diagnosed with substance abuse / dependence admitted to an Intensive Care Unit. Method: retrospective, descriptive-exploratory, cross-sectional and quantitative study. Results: a total of 865 medical records were analyzed between 2012 and 2015. The prevalence of substance use was 51.9\%, of these $22 \%$ of total alcohol users and $48.7 \%$ tobacco. Among the users with diagnosed dependence, the main causes of hospitalization were Chronic Obstructive Pulmonary Disease $(100 \%)$ and digestive hemorrhage $(84 \%)$ and most patients presented more than one comorbidity simultaneously. Conclusion: the results showed numerous causes of hospitalization and comorbidities that demonstrated the physical and mental impairment that may be related to excessive consumption of psychoactive substances.

Descriptors: Substance-Related Disorders; Drug Users; Intensive Care Unit; Comorbidities.

\section{Características clínicas de consumidores de drogas internados en unidad de terapia intensiva}

Objetivo: describir las causas de internación y comorbilidades de los pacientes con diagnóstico de abuso / dependencia de sustancias ingresados en una Unidad de Cuidados Intensivos. Método: estudio retrospectivo, descriptivo-exploratorio, transversal y cuantitativo. Resultados: se examinaron 865 registros médicos entre 2012 y 2015, la prevalencia de uso de sustancias fue de $51.9 \%$, de estos $22 \%$ consumidores de alcohol y $48.7 \%$ de tabaco. Entre los usuarios con dependencia diagnosticada, las principales causas de hospitalización fueron la enfermedad pulmonar obstructiva crónica (100\%) y la hemorragia digestiva (84\%) y la mayoría de los pacientes presentaron más de una comorbilidad simultáneamente. Conclusión: los resultados mostraron numerosas causas de hospitalización y comorbilidades que demostraron el deterioro físico y mental que puede estar relacionado con el consumo excesivo de sustancias psicoactivas.

Descriptores: Trastornos Relacionados con Sustancias; Consumidores de Drogas; Unidad de Terapia Intensiva; Comorbilidades. 


\section{Introdução}

O uso abusivo de álcool e outras drogas representa grave problema de saúde pública, pois pode progredir de forma lenta e insidiosa, evoluindo para a cronificação, acarretando problemas graves no processo saúdedoença do indivíduo, família e sociedade ${ }^{(1)}$.

O impacto do uso abusivo de drogas para o conjunto da sociedade é incalculável em todos os países, pois se relaciona à possibilidade de comorbidades, mortalidade precoce, incremento da violência e criminalidade, acidentes de trânsito e de trabalho, absenteísmo, distúrbios emocionais, conflitos familiares e sociais ${ }^{(2)}$.

O consumo de álcool, tabaco e outras drogas lícitas está aumentando e contribuindo de maneira evidente para a carga de doenças em todo o mundo. A maior parte dos problemas mundiais decorrentes do consumo de substâncias psicoativas provém das drogas lícitas. O tabaco e o álcool aparecem como importantes causas de mortalidade e incapacidade nos países desenvolvidos e importantes fatores de risco, em termos de carga de enfermidades evitáveis, na América Latina. O consumo de álcool é a terceira causa de doença e morte prematura em nível mundial, 3,3 milhões de mortes e 5,1\% da carga global de doenças ${ }^{(3-4)}$.

A assistência a usuários de álcool e outras drogas deve se dar em todos os níveis de atenção, privilegiando os dispositivos extra-hospitalares, porém, nos casos de intercorrências clínicas, as instituições hospitalares são locais estratégicos na rede de atenção à saúde. Quando criticamente enferma, essa população pode necessitar de acesso à internação em Unidades de Terapia Intensiva (UTI).

Diante do exposto e da escassez de estudos a respeito do tema, esta pesquisa tem por objetivo descrever as causas de internação, comorbidades e características clínicas dessa população, quando internada em Unidade de Terapia Intensiva.

\section{Método}

Estudo retrospectivo, de caráter descritivoexploratório, transversal e quantitativo, utilizando fonte secundária de dados, realizado em hospital de ensino da cidade de Pelotas - Rio Grande do Sul, o qual atende exclusivamente usuários do Sistema Único de Saúde, especificamente em UTI. A UTI estudada é composta por seis leitos, recebe pacientes clínicos e cirúrgicos, excluindo internações por trauma, cirurgias cardíacas e neurológicas.

Para o cálculo da amostra, com base na literatura, estimou-se que a prevalência de abuso de álcool e outras drogas em pacientes internados em UTI é de $25 \%$, já que foi o estudo que mais se assemelhou em relação à população investigada e metodologia. Com erro tolerável de \pm 3 pontos percentuais e nível de confiança de $95 \%$, calculou-se um tamanho de amostra de 880 pacientes, utilizando o software EPI INFO(5).

A fim de atingir o tamanho da amostra estimado, verificou-se, mediante contagem, o número de internações retrospectivamente, utilizando-se o livro de registro de internações da unidade. Inicialmente, estimou-se que, para atingir o tamanho da amostra, seriam necessários cinco anos de levantamento e um número médio de internações anuais de 200 usuários, porém, o número foi atingido em quatro anos de levantamento.

Foi elaborada tabela com o número de prontuário de todos os pacientes internados na UTI, no período de 2012 a 2015, levantados do livro de registro de internação da unidade. Na sequência, foi realizada a busca dos dados no Setor de Arquivo Médico e Estatística (SAME). Apleicou-se um instrumento pré-elaborado com questões fechadas e codificadas.

A identificação dos prontuários e a coleta dos dados foram realizadas pela autora do projeto. Foram pesquisados 30 prontuários por turno de coleta, durante três dias da semana, contemplando 90 prontuários por semana, atingindo a amostra proposta em aproximadamente dez semanas, ao longo dos meses de julho, agosto e setembro de 2016.

A variável dependente correspondeu ao "diagnóstico de abuso/dependência de álcool e outras drogas". As variáveis independentes foram sexo, idade, estado civil, ocupação, tipo de substância utilizada, comorbidades, tempo de ventilação mecânica, causa da internação, tempo de internação na UTI e desfecho da internação.

Os dados coletados foram digitados no MS Access (Microsoft Office Access). Realizaram-se, inicialmente, análises exploratórias, visando a caracterizar a população de estudo e a responder aos objetivos propostos mediante o uso de medidas descritivas (média, moda, mediana) e de dispersão (desvio-padrão).

A segunda fase foi a verificação de associações entre o diagnóstico de abuso/uso de álcool e outras drogas (desfecho) e variáveis independentes por meio da aplicação dos testes qui-quadrado de Pearson ou o exato de Fisher (frequências $<5$ ).

Posteriormente, foi estimada a magnitude das associações. Utilizou-se a prevalência de pacientes internados com diagnóstico de uso abusivo de álcool e outras drogas e, como medida de associação, a Razão de Prevalência (RP) e seus respectivos intervalos de confiança a $95 \%$, estimados em função da incidência relativa da Regressão de Poisson por meio do método de variância robusta. Adotou-se o nível de significância estatístico de $5 \%(a \leq 0,05)$.

O estudo observou o Código de Ética dos Profissionais de Enfermagem, embasado na Resolução 
COFEN 311/2007, Cap. III, Art. 89, 90 e 91, e na Resolução no 466/2012 do Conselho Nacional de Saúde, sendo aprovado pelo Comitê de Ética em Pesquisa da Faculdade de Medicina da Universidade Federal de Pelotas sob o parecer n 1.540 .724 .

\section{Resultados}

Foram identificados 877 prontuários de pacientes admitidos na Unidade de Terapia Intensiva (UTI) durante o período de 2012 a 2015. Do total, 12 prontuários foram excluídos por não ter sido localizados no serviço de arquivamento e representaram $1,7 \%$ do total no período, permanecendo 865 prontuários $(n=865)$.

No período delimitado, a prevalência de pacientes internados com diagnóstico de uso abusivo de álcool e outras drogas foi de $51,9 \%(n=449)$. A média de idade dos pacientes diagnosticados como dependentes de álcool e outras drogas foi de 59,3 3 15,31 anos e o sexo masculino foi predominante $(68,9 \%)$ entre os dependentes, enquanto entre os não dependentes predominou o sexo feminino $(65,1 \%)$.

A prevalência, em relação à população total de internados na UTI, de usuários de álcool é de $22 \%$; de tabaco, 48,7\%; de maconha, 3\%; de cocaína, 2,3\%; de crack, $2,7 \%$ e de outras drogas, $0,1 \%$, sendo que $22 \%$ do total de internados eram usuários de múltiplas drogas. O tempo médio de consumo de álcool foi de 26,3 $\pm 10,2$ anos; o de tabaco foi de 35,6 \pm 13,2 anos; o de maconha foi de 15,4 $\pm 6,2$ anos; o de cocaína, 14,5 \pm 6,9 anos e o de crack, de 9,6 \pm 4,7 anos.

Abaixo, apresenta-se a Tabela 1 referente à distribuição proporcional da causa da internação dos pacientes admitidos na UTI.

Tabela 1 - Distribuição proporcional da causa da internação dos pacientes admitidos em uma Unidade de Terapia Intensiva $(n=865)$. Pelotas, RS, Brasil, 2016

\begin{tabular}{|c|c|c|c|c|c|}
\hline \multirow{3}{*}{ Causa da Internação } & \multicolumn{4}{|c|}{ Diagnóstico de uso abusivo de álcool e outras drogas } & \multirow{3}{*}{ p-valor } \\
\hline & \multicolumn{2}{|c|}{ Dependente } & \multicolumn{2}{|c|}{ Não Dependente } & \\
\hline & $\mathbf{n}$ & $\%$ & $\mathbf{n}$ & $\%$ & \\
\hline DPOC & 22 & 100 & 0 & 0 & 0,000 \\
\hline Hemorragia digestiva & 21 & 84,0 & 4 & 16,0 & 0,001 \\
\hline Infarto agudo do miocárdio & 4 & 66,7 & 2 & 33,3 & 0,688 \\
\hline Insuficiência hepática & 6 & 66,7 & 3 & 33,3 & 0,508 \\
\hline Outras cardiopatias & 7 & 63,6 & 4 & 36,4 & 0,550 \\
\hline Insuficiência respiratória aguda & 142 & 60,7 & 92 & 39,3 & 0,002 \\
\hline Pneumonia & 30 & 62,5 & 18 & 37,5 & 0,140 \\
\hline Complicações do $\mathrm{HIV}^{\dagger} /$ Aids $^{\ddagger}$ & 22 & 59,5 & 15 & 40,5 & 0,402 \\
\hline Sepse & 128 & 50,8 & 124 & 49,2 & 0,708 \\
\hline Pós-operatório não oncológico & 24 & 49 & 25 & 51 & 0,769 \\
\hline Pós-operatório oncológico & 80 & 46,8 & 91 & 53,2 & 0,147 \\
\hline Acidente vascular cerebral & 11 & 45,8 & 13 & 54,2 & 0,680 \\
\hline Insuficiência renal aguda & 20 & 45,4 & 24 & 54,6 & 0,440 \\
\hline Complicações oncológicas & 52 & 42,3 & 71 & 57,7 & 0,025 \\
\hline Outros & 91 & 45,7 & 108 & 54,3 & 0,052 \\
\hline
\end{tabular}

*Teste Exato de Fischer; ${ }^{+\neq}$

Em relação às comorbidades dos pacientes internados na UTI, a maioria dos pacientes apresentou mais do que uma comorbidade simultaneamente. Dos que apresentavam comorbidades cardiovasculares, os usuários com diagnóstico de uso abusivo de álcool e outras drogas eram 47,4\%; respiratórias, 72,7\%; infecciosas, $60,3 \%$; neoplasias, $51,8 \%$; neurológicas, $32,1 \%$; renais ou metabólicas, 35,3\%; gastrointestinais, $78,1 \%$ e psiquiátricas, $31,2 \%$.

Dentre as principais comorbidades cardiovasculares, destacam-se a hipertensão arterial sistêmica, o infarto agudo do miocárdio e as arritmias. Das respiratórias, as principais foram a insuficiência respiratória aguda, a DPOC e o edema agudo de pulmão. Dentre as infecciosas, sepse, pneumonias, SIDA e infecções oportunistas. Dentre as neoplasias, as principais foram as do aparelho digestório, hematológicas e pulmonares. Das comorbidades neurológicas, a principal foi o acidente vascular cerebral. Dentre as renais ou metabólicas, as principais foram o Diabetes Mellitus, a insuficiência renal aguda e crônica e a cetoacidose diabética. Das gastrointestinais, as principais foram a hemorragia digestiva alta e a cirrose hepática e, das comorbidades psiquiátricas, as mais presentes foram a depressão, a ansiedade e o transtorno afetivo bipolar.

Na tabela 2, avaliou-se a magnitude das associações entre admissões em UTI de pacientes dependentes comparados aos não dependentes segundo as características clínicas. 
Tabela 2 - Associação entre pacientes admitidos em uma Unidade de Terapia Intensiva e as características clínicas $(n=865)$. Pelotas, RS, Brasil, 2016

\begin{tabular}{|c|c|c|c|}
\hline Característica & $\begin{array}{l}\text { Total } \\
\text { n (\%) }\end{array}$ & $\begin{array}{c}\text { Prevalência } \\
\text { Pacientes com uso abusivo de álcool e } \\
\text { outras drogas } \\
\mathrm{n}(\%)\end{array}$ & $\mathrm{RP}_{\text {Bruta }}^{*}$ (IC 95\%) \\
\hline \multicolumn{4}{|l|}{ Desfecho internação em UTI } \\
\hline Alta para enfermaria & $433(50,1)$ & $210(48,5)$ & $1,0^{\dagger}$ \\
\hline Óbito & $408(47,2)$ & $228(55,9)$ & $1,2(1,0-1,2)$ \\
\hline Transferência de UTI & $24(2,8)$ & $11(45,8)$ & $0,9(0,6-1,5)$ \\
\hline \multicolumn{4}{|l|}{ Tempo de internação } \\
\hline$<3$ dias & $282(32,6)$ & $122(43,6)$ & $1,0^{+}$ \\
\hline 3 a 7 & $298(34,4)$ & $167(55,8)$ & $1,3(1,1-1,5)$ \\
\hline 8 a 15 & $146(16,9)$ & $77(52,7)$ & $1,2(1,0-1,5)$ \\
\hline 16 a 20 & $36(4,2)$ & $26(72,2)$ & $1,7(1,3-2,1)$ \\
\hline 21 a 30 & $55(6,4)$ & $30(53,6)$ & $1,2(0,9-1,6)$ \\
\hline 31 e mais & $48(5,6)$ & $27(56,2)$ & $1,3(1,0-1,7)$ \\
\hline \multicolumn{4}{|l|}{ Uso de ventilação mecânica } \\
\hline Não & $357(41,3)$ & $162(45,4)$ & $1,0^{+}$ \\
\hline Sim & $508(58,7)$ & $287(56,5)$ & $1,2(1,1-1,4)$ \\
\hline \multicolumn{4}{|l|}{ Duração da VM ( $n=507$ ) } \\
\hline$<3$ dias & $177(34,9)$ & $86(48,6)$ & $1,0^{+}$ \\
\hline 3 a 7 & $156(30,8)$ & $92(59,0)$ & $1,2(1,0-1,5)$ \\
\hline 8 a 15 & $88(17,4)$ & $55(62,5)$ & $1,3(1,0-1,6)$ \\
\hline 16 a 20 & $21(4,1)$ & $14(66,7)$ & $1,4(1,0-1,9)$ \\
\hline 21 a 30 & $35(6,9)$ & $21(60,0)$ & $1,2(0,9-1,7)$ \\
\hline 31 e mais & $30(5,9)$ & $19(63,3)$ & $1,3(1,0-1,8)$ \\
\hline
\end{tabular}

*Razão de Prevalência: estimada em função do risco relativo da Regressão de Poisson robusta; ${ }^{+}$Categoria de referência

\section{Discussão}

A utilização abusiva de substâncias psicoativas está relacionada a prejuízos à saúde mental e física dos usuários e, dentre as principais consequências físicas, encontram-se os problemas hepáticos, problemas relacionados à síndrome de abstinência, prejuízos do sono e distúrbios gastrointestinais ${ }^{(2)}$.

Em relação às causas de internação, o estudo demonstrou que $100 \%$ dos pacientes internados com DPOC possuíam diagnóstico de uso abusivo ou dependência de drogas, o que pode estar relacionado à alta prevalência encontrada de usuários de tabaco internados em UTI $(48,7 \%)$. As doenças relacionadas ao tabaco são uma das principais causas de internação hospitalar, especialmente em idosos, e a redução do tabagismo contribui para a diminuição da morbidade e da mortalidade(6).

Tabaco, álcool e drogas ilícitas estão entre os principais 20 fatores de risco para doenças. Tal fato decorre do encargo global devido às doenças associadas ao uso do tabaco (anos de incapacidade de adaptação da vida- DALY). Estima-se que o tabaco seja responsável por $8,8 \%$ de todas as mortes e por $4,1 \%$ da $\operatorname{DALY}(7)$.
O tabagismo responde atualmente por $45 \%$ de todas as mortes por câncer, 95\% das mortes por câncer de pulmão, $75 \%$ das mortes por DPOC e $35 \%$ das mortes por doenças cardiovasculares ${ }^{(6)}$.

O estudo demonstrou que $22 \%$ dos pacientes com diagnóstico de uso abusivo ou dependência de álcool e outras drogas eram usuários de múltiplas drogas. Preditores psiquiátricos que incentivam o início do uso do cigarro incluem o consumo abusivo de álcool e de outras drogas psicoativas, bem como a presença de transtornos de déficit de atenção e de sintomas depressivos ${ }^{(4)}$.

Evidenciou-se que $84 \%$ das internações por hemorragia digestiva e $66,7 \%$ das internações por insuficiência hepática eram de pacientes com diagnóstico de uso abusivo ou dependência de álcool e outras drogas. Estudo irlandês encontrou, entre 170 pacientes internados em UTI por doença hepática, 62 dependentes de álcool (36,5\%). No Reino Unido, a doença hepática constitui a terceira causa mais comum de morte prematura, e mais de um milhão de internações hospitalares por ano são resultado de distúrbios relacionados ao álcool(7-8).

O consumo excessivo de álcool, conforme a literatura, mantém relação causal com mais de 200 tipos de doenças e lesões, podendo ser a principal 
causa de hospitalização. O consumo do álcool aumenta o risco do desenvolvimento ou está associado aos agravos de várias patologias, como câncer, hipertensão, doença cerebrovascular, infarto agudo do miocárdio, doenças do fígado, tuberculose, diabetes, cirrose hepática, osteoporose, HIV/Aids, além de complicações psiquiátricas e casos de agressões com graves consequências ${ }^{(9)}$.

Dos pacientes internados na UTI por complicações relacionadas ao $\mathrm{HIV} / \mathrm{Aids}, 59,5 \%$ possuíam o diagnóstico de uso abusivo de álcool e outras drogas, prevalência semelhante à encontrada na literatura em estudo canadense, que foi de $56 \%$. O uso de drogas injetáveis, como cocaína e opioides, pode provocar doenças infecciosas e inflamatórias, locais ou disseminadas, e está muito associado ao contágio pelos vírus da imunodeficiência humana (HIV) e das hepatites por meio do compartilhamento de seringas. Nesta pesquisa, não se identificou a utilização de heroína injetável, porém, houve diversos registros de cocaína utilizada por via endovenosa(10-11).

Das internações por sepse, 50,8\% eram diagnosticados com uso abusivo ou dependência de álcool e outras drogas. A literatura confirma a relação entre sepse e dependência de álcool a partir de estudo estadunidense que pesquisou 11651 prontuários de internações em UTI no período de cinco anos e obteve uma prevalência de $12 \%$ de dependentes de álcool, menor do que a deste estudo. Esses pacientes apresentaram maiores taxas de sepse $(12,9 \%$ vs $7,6 \%$, $\mathrm{p}<0,001)$, falência de órgãos $(67,3 \%$ vs $45,8 \%$, $\mathrm{p}<0,001)$, choque séptico $(3,6 \%$ vs $2,1 \%, \mathrm{p}=0,01)$ e mortalidade hospitalar $(9,4 \%$ vs $7,5 \%, p=0,022)$ em análises não ajustadas. Após o ajuste para fatores com associação conhecida com sepse, a dependência de álcool manteve associação com sepse, choque séptico e mortalidade hospitalar ${ }^{(11)}$.

Importante discutir a questão do diagnóstico de uso de álcool e outras drogas já que, na unidade investigada, não se utiliza escala diagnóstica validada e a informação é bastante atrelada ao relatado por pacientes e familiares. A estratégia de avaliar o consumo somente a partir do relato do entrevistado possivelmente resulta na ocorrência de viés de informação decorrente da omissão do consumo abusivo pelos entrevistados, o que possivelmente resulta em subestimação das prevalências ${ }^{(4)}$.

A presença de comorbidades cardiovasculares é condizente com o resultado de outras pesquisas que associam o consumo de substâncias psicoativas e alterações desse sistema, sendo o álcool ao aumento da pressão arterial, desregulação de triglicérides e lipídeos, maior risco de infarto agudo do miocárdio e outras doenças cardiovasculares. Estudo realizado no
Brasil observou uma prevalência de $25 \%$ de hipertensos entre pacientes CAGE (acrônimo referente às suas quatro perguntas - Cut down, Annoyed by criticism, Guilty e Eye-opener) positivo. A hipertensão tem acometimento multifatorial, porém, acredita-se que o consumo excessivo de álcool é o segundo fator de risco não genético para a doença(9-13).

Evidenciou-se prevalência importante de comorbidades gastrointestinais $(78,1 \%)$ entre os internados com diagnóstico de uso abusivo ou dependência de álcool e outras drogas provavelmente relacionadas ao uso excessivo de álcool. Pesquisa realizada com pacientes internados em unidade de internação clínica com diagnóstico de etilismo encontrou prevalência de $77,8 \%$ de hepatite alcoólica, $60 \%$ de cirrose hepática e $26,7 \%$ de hemorragia digestiva alta. Acredita-se que cerca de $35 \%$ dos pacientes cirróticos com varizes esofágicas ou gástricas sangrarão no futuro e mais de $20 \%$ deles morrerão no primeiro episódio( ${ }^{(9)}$.

Em relação às comorbidades psiquiátricas, o estudo corrobora outros que demonstram que os transtornos de humor, como a depressão, uni ou bipolar, e os transtornos de ansiedade são prevalentes entre as comorbidades psiquiátricas associadas à dependência química, sendo menos frequentes as patologias com sintomas psicóticos. Estudo realizado em comunidades terapêuticas observou prevalência elevada de comorbidades psiquiátricas $(88,8 \%)$ e, em específico, do transtorno depressivo maior $(36,7 \%)$ e do transtorno de personalidade antissocial $(76,7 \%)^{(12)}$.

Verificou-se a gravidade dos pacientes internados na UTI com diagnóstico de uso abusivo ou dependência de álcool e outras drogas, já que a chance do desfecho de internação óbito ocorrer é 1,2 vezes superior (IC 95\%:1,0-1,2); de apresentarem internações prolongadas, com duração de 15 a 20 dias, é 1,7 vezes superior (IC 95\%: 1,3-2,1) e 1,2 vezes superior de fazer uso de ventilação mecânica (IC 95\%: 1,1-1,4), quando comparados aos não dependentes.

A média temporal de permanência em UTI varia entre quatro a dez dias, e a demora no período de estada na UTI associa-se ao mau prognóstico na recuperação do paciente, bem como a custos econômicos elevados. A VM é tratamento frequente e proporciona inúmeros benefícios para o tratamento de pacientes críticos, porém, quanto maior o tempo de VM, maior é o tempo de internação, aumentando também os riscos de complicações ${ }^{(14-15)}$.

Torna-se importante mencionar que este estudo demonstra uma face do problema de saúde pública, que é o uso abusivo de drogas psicoativas, e este possui ligação direta ou indireta com os determinantes sociais e, neste contexto, necessita de políticas públicas 
resolutivas que possam fortalecer as condições sociais, econômicas e de saúde dos usuários e da coletividade ${ }^{(16)}$.

Os resultados e a literatura demonstraram inúmeras causas de internação e comorbidades clínicas e psiquiátricas que podem estar associadas ao uso excessivo de álcool e outras drogas em pacientes que necessitam de cuidados intensivos.

\section{Considerações finais}

Esta pesquisa mostrou as principais causas de internação, comorbidades mais frequentes e características clínicas de pacientes internados em Unidade de Terapia Intensiva com diagnóstico de uso abusivo ou dependência de álcool e outras drogas, demonstrando comprometimento físico e psíquico que pode estar relacionado ao consumo excessivo de substâncias psicoativas.

Evidenciou-se que, por diversas patologias e evolução negativa delas, os pacientes que fazem uso excessivo de álcool e outras drogas podem chegar a situações de criticidade e necessitar de internação em UTI.

As limitações do estudo deram-se em relação aos registros em prontuários, por déficit de informações dos padrões de uso e do diagnóstico de transtornos relacionados ao uso de substâncias, já que não existe padronização de escala diagnóstica. A pesquisa colaborou para conhecer a questão do uso abusivo de substâncias psicoativas durante a internação em UTI, a gravidade das patologias que os acometem, para que se possa ofertar atendimento integral aos usuários. Ainda veio preencher uma lacuna de conhecimento, pois há escassa produção sobre a temática.

Ao conhecer essa realidade, a equipe de saúde pode preparar-se para atender não somente à causa de internação e às principais comorbidades, como valorizar o uso de substância psicoativa para planejar melhor a terapêutica, prestar orientações aos pacientes e familiares e encaminhar adequadamente o usuário dentro da rede de atenção à saúde.

\section{Referências}

1. Nascimento LTR, Souza J, Gaino LV. Relacionamento entre familiar e usuário de álcool em tratamento em um centro de atenção psicossocial especializado. Texto Contexto Enferm. 2015; 24(3): 834-41. doi: http:// dx.doi.org/10.1590/0104-07072015003610013

2. Silva ER, Ferreira ACZ, Borba LO, Kalinke LP, Nimtz MA, Maftum MA. Impacto das drogas na saúde física e mental de dependentes químicos. Cienc Cuidado Saúde. 2016 Jan/Mar; 15(1):101-8. doi: http://dx.doi. org/10.4025/cienccuidsaude.v15i1.27137

3. Barbosa MB, Pereira CV, Cruz DT, Leite ICG. Prevalência e fatores associados ao consumo de álcool e de tabaco em idosos não institucionalizados. Rev Bras Geriatr Gerontol. 2018;21(2):125-35. http://dx.doi. org/10.1590/1981-22562018021.170185

4. Garcia LP, Freitas LRS. Consumo abusivo de álcool no Brasil: resultados da Pesquisa Nacional de Saúde 2013. Epidemiol Serv Saúde. 2015; 24(2): 227-237. doi: http://dx.doi.org/10.5123/S1679-49742015000200005 5. Geary T, O'Brien P, Ramsay S, Cook B. Scottish Intensive Care Trainees Audit Share Group. A national servisse evaluation of the impacto of alcohol on admissions to Scottish Intensive Care Units. Anaesthesia. 2012 Oct; 67 (10): 1132-7. doi: https:// doi.org/10.1111/j.1365-2044.2012.07233.x

6. Marques AP, Montilla DER, Almeida WS, Andrade CLT. Internação de idosos por condições sensíveis a atenção primária à saúde. Rev Saúde Pública. 2014;48(5): 819-26. https://doi.org/10.1590/ S0034-8910.2014048005133

7. Fröhlich S, Murphy N, Kong T, Ffrench-O'Carroll $R$, Conlon N, Ryan D et al. Alcoholic liver Disease in the intensive care unit - outcomes and predictors of prognosis. J Crit Care. 2014; 29 (6): 1137.e7-1131.e13. doi: https://doi.org/10.1016/j.jcrc.2014.06.002

8. Williams R, Aspinall R, Bellis M, Camps-Walsh G, Cramp $M$, Dhawan $A$, et al. Addressing liver disease in the UK: a blueprint for attaining excellence in health care and reducing premature mortality from lifestyle issues of excess consumption of alcohol, obesity, and viral hepatites. Lancet. 2014;384: 1953-97. https://doi. org/10.1016/S0140-6736(14)61838-9

9. OPAS Brasil - Organização Pan-Americana da Saúde. Folha informativa - álcool. Brasília; 2019. [Acesso 5 jul 2019]. Disponível em: https://www.paho.org/bra/ index.php?option $=$ com_content $\&$ view $=$ article\&id $=5649$ : folha-informativa-alcool\&Itemid $=1093$

10. Santos RB. Prevalência e fatores associados à infecção pelo hiv entre usuários de drogas lícitas e ilícitas. Dissertação [Mestrado Ciências da Saúde]. Recife: Universidade Federal de Pernambuco; 2014. URL: https://repositorio.ufpe.br/handle/123456789/17744

11. O'Brien JM Jr, Lu B, Ali NA, Martin GS, Aberegg SK, Marsh $C B$ et al. Alcohol dependence is independently associated with sepsis, septic shock and hospital mortality among adult intensive care unit patients. Crit Care Med. 2007; 35 (2): 345-50. doi: https://doi. org/10.1097/01.CCM.0000254340.91644.B2

12. Danieli RV, Ferreira MBM, Nogueira JM, Oliveira LNC, Cruz EMTN, Araújo Filho GM. Perfil sociodemográfico e comorbidades psiquiátricas em dependentes químicos acompanhados em comunidades terapêuticas. J Bras Psiquiatr. 2017;66(3):139-49. doi: 10.1590/0047-2085000000163

13. Capistrano FC, Ferreira ACZ, Silva TL, Kalinke LP, Maftum MA. Perfil sociodemográfico e clínico de dependentes químicos em tratamento: análise de 
prontuários. Esc Anna Nery. 2013; 17 (2):234-41. doi: http://dx.doi.org/10.1590/S1414-81452013000200005 14. Sousa MNA, Cavalcante AM, Sobreira REF, Bezerra ALD, Assis EV, Feitosa ANA. Epidemiologia das internações em unidade de terapia intensiva. C\&D Rev Eletrôn Fainor. (Vitória da Conquista). 2014;7(2):178-86. Disponível em: http://srv02.fainor.com.br/revista/index. php/memorias/article/view/288

15. Murakami FM, Yamaguti WP, Onoue MA, Mendes JM, Pedrosa RS, Maida ALV et al. Evolução funcional de pacientes graves submetidos a um protocolo de reabilitação precoce. Rev Bras Ter Intensiva. 2015;27(2):161-9. Disponível em: http://www.scielo. br/pdf/rbti/v27n2/0103-507X-rbti-27-02-0161.pdf

16. Ventura CAA. Determinantes Sociais de Saúde e o uso de drogas psicoativas. SMAD - Revista Eletrônica Saúde Mental Álcool e Drogas. 2014; 10(3): 110. doi:http://dx.doi.org/10.11606/issn.1806-6976. v10i3p110-110

\section{Contribuição dos autores}

Concepção e planejamento do estudo: Gabriela Botelho Pereira, Michele Mandagará de Oliveira. Obtenção dos dados: Gabriela Botelho Pereira, Michele Mandagará de Oliveira. Análise e interpretação dos dados: Gabriela Botelho Pereira, Karine Langmantel Silveira, Carla Luciane dos Santos Borges, Michele Mandagará de Oliveira. Preparação e rredação do manuscrito: Gabriela Botelho Pereira, Carla Luciane dos Santos Borges. Revisão crítica do manuscrito: Carla Luciane dos Santos Borges, Michele Mandagará de Oliveira.

Todos os autores aprovaram a versão final do manuscrito.

Conflito de interesse: Os autores declaram não haver conflito de interesse
Recebido: 30.05 .2019

Aceito: 26.07.2019
Autor correspondente:

Gabriela Botelho Pereira

E-mail: enfagabi@bol.com.br

(D) https://orcid.org/0000-0002-9964-6586
Copyright $\odot 2020$ SMAD, Rev. Eletrônica Saúde Mental Álcool Drog. Este é um artigo de acesso aberto distribuído sob os termos da Licença Creative Commons CC BY-NC.

Esta licença permite que outros remixem, adaptem e criem a partir do seu trabalho para fins não comerciais, e embora os novos trabalhos tenham de lhe atribuir o devido crédito e não possam ser usados para fins comerciais, os usuários não têm de licenciar esses trabalhos derivados sob os mesmos termos. 